\title{
Evaluación de la educación digital y digitalización de la evaluación
}

\section{(Assessing Online Learning and the Digitalization of Assessment)}

\author{
Elena Barberà-Gregori \\ Universitat Oberta de Catalunya (España) \\ Cristóbal Suárez-Guerrero \\ Universitat de València (España) \\ Coordinadores del Monográfico
}

DOI: https://doi.org/10.5944/ried.24.2.30289

\section{Cómo referenciar este artículo:}

Barberà-Gregori, E., y Suárez-Guerrero, C. (2021). Evaluación de la educación digital y digitalización de la evaluación. RIED. Revista Iberoamericana de EducaciónaDistancia,24(2),pp.33-40.https://doi.org/10.5944/ried.24.2.30289

\section{Resumen}

La evaluación de la educación digital y digitalización de la evaluación son procesos por el que transita tanto la educación digital como la educación digital de emergencia. Aunque el tema de la evaluación, por sí misma, ya es complejo, esta complejidad aumenta cuando media la tecnología digital. Lejos de pensar que se trata de una acción técnica, la evaluación digital y la digitalización de la evaluación, como lo demuestra el contenido de este monográfico, no se reducen a la inserción de sistemas tecnológicos, sino a la comprensión pedagógica de su rol e impacto en los procesos educativos. Los trabajos que conforman este monográfico buscan contribuir, desde el análisis empírico y la reflexión pedagógica, a la construcción de una irremediable línea de trabajo que se pregunta: ¿cómo transforma, y transformamos, la condición digital, procesos tan sensibles como la evaluación del aprendizaje mediado y la evaluación de los sistemas educativos digitales? Al respecto, en este número se avanza en la respuesta en torno a cuatro núcleos. Por un lado, trabajos que ponen énfasis en la nueva exigencia digital impuesta a la evaluación en tiempos de pandemia, el papel renovado del feedback educativo en la necesaria autorregulación con soporte tecnológico, la necesidad de encarar con garantías la evaluación digital como condición de rendimiento y, como mecanismo observante, la evaluación de la calidad de la educación digital. Estos trabajos sobre evaluación educativa tienen poco de simple, mucho de complejo y bastante de complicado, pero esta es su naturaleza y con la que hay que entender el hecho digital. 
Palabras clave: aprendizaje; evaluación; tecnología de la educación; tecnologías de la información y la comunicación; innovación pedagógica.

\begin{abstract}
Assessment and its digitalization are key elements of both online learning and emergency remote teaching. On its own, assessment is a complex subject, but add digital technology and this complexity increases tenfold. Far from a mechanical action, the assessment of online learning and the digitalization of this process involve more than just the incorporation of technological systems; as this special issue will show, they are concepts whose role in and impact on teaching must be understood at a pedagogical level. Based on empirical analysis and pedagogical reflection, the papers in this special issue seek to construct an irrefutably important line of work that poses the question, how does the digital environment transform sensitive processes such as ICT-mediated learning assessment and the assessment of online learning systems, and how do we in turn transform it? The issue tackles this question from four different angles: by highlighting the new digital demands placed on assessment in times of pandemic; the renewed role of educational feedback in the ICT-based self-regulated learning required; the need to move towards e-assessment as a guarantee of performance, and the evaluation of the quality of online learning as a watchdog mechanism. As the special issue on learning assessment shows, the topic is full of challenges, but that is the nature of the beast, so to speak, and how one must approach all things digital.
\end{abstract}

Keywords: learning; assessment; education technology; information and communication technologies; pedagogical innovation.

Al escribir este artículo es imposible dejar de sopesar el cambio inédito y global que toda la humanidad encara hoy. Los cambios que impone, y los que llegarán, no son aspectos accesorios, sino que esta pandemia por COVID-19 está generando el replanteamiento de nada más y nada menos que nuestras relaciones sociales. ¿̇Y qué es la educación en esencia sino una práctica social, básica, sustancial y aquella por la que nos humanizamos? Alojados en este cambio de época impuesto por esta pandemia, estamos tomando, como nunca, una serie de decisiones basadas en procesos de evaluación cada vez más constantes, más fundados, pero no por ello alejados de incertidumbre, que a su vez guían muchos procesos como la propia salud, la conducta social, la gestión del estado, la dinámica económica y más aspectos de la vida diaria. La educación, específicamente la educación formal, como no se trata de una entidad abstracta, ha sido presa de los cambios y, en general, por ensayo y error, hemos diseñado de manera muy rápida, una educación digital de emergencia (Hodges et al., 2020). No obstante, esta forma de educar propia de la pandemia no es la educación digital que de forma sistemática y empírica viene, desde hace tres décadas, creando otros escenarios de educación, especialmente en la educación universitaria. La educación digital de emergencia no sería la educación digital en 
sí misma, esta es una primera valoración que hay que tener en cuenta respecto a la digitalización de los procesos de evaluación educativa.

Aunque la educación digital de emergencia no sea la educación digital propiamente dicha es, sin duda, parte de la actual realidad educativa. Lo digital, para muchos sectores de la vida, ha representado una salida a la crisis, pero no la solución. Los problemas estructurales de la educación y la escuela siguen ahí, es más, en cierto modo han aumentado por la crisis. Sin embargo, la crisis sanitaria está dejando en evidencia, acentuada en la conciencia colectiva, que la condición digital ya forma parte ineludible de nuestro imaginario educativo y que es tiempo de encarar una reflexión sobre su mejora e impacto. Este monográfico forma parte de esta línea de reflexión inexorable sobre cómo transforma, y transformamos, la condición digital de la evaluación tanto del aprendizaje mediado, así como de los sistemas educativos digitales.

Ahora bien, todos tenemos una idea más o menos certera sobre cómo o qué debe ofrecer una universidad y una clase presencial, pero, ¿qué ocurre cuando se digitaliza?, ¿cuáles son esos mínimos, los criterios o las expectativas, que deben de cumplir la experiencia de aprendizaje digital o el sistema educativo digital? La situación ha llevado a atender, desde distintas miradas y ritmos de trabajo, cambios y aceptar la digitalización de la experiencia universitaria como parte de una nueva normalidad.

Por ejemplo, la Agencia Nacional de Evaluación de la Calidad y Acreditación (ANECA) de España, a través de diversas directrices, ha tenido que acelerar sus respuestas y su comprensión sobre la nueva educación digitalizada. En este proceso, la exigencia evaluativa ha sido la constante: ¿cómo cumplir con un estándar universitario cuando el contexto donde se desarrollaba ha desaparecido y ha sido reemplazado por una interfaz digital? En un documento publicado al inicio de la pandemia, la ANECA señalaba sobre la evaluación del aprendizaje que "parece adecuado optar por métodos alternativos al examen único: evaluación continua, seguimiento directo, trabajos individuales o en grupo, entrevistas de tú a tú, etc." (2020, p. 8) y, señalaba que una de las claves de la salvaguardia de la calidad de los modelos online y modelos mixtos de aprendizaje, en tiempos de pandemia residían no tanto en la revisión externa, sino en la evaluación de los sistemas internos, la autoevaluación. Esto es, lo digital, tanto en la educación como especialmente en la praxis evaluativa, no es un asunto técnico o solo de herramientas, implica cambios en la forma de pensar la educación, sus expectativas, sus metas, su alcance, su impacto.

La evaluación de la educación digital y digitalización de la evaluación, por tanto, requiere marcos de comprensión renovados, con o sin pandemia. En este marco de modernización de la evaluación en ámbitos educativos emerge también, en los últimos años, el uso de ciertos conocimientos que provienen de otras disciplinas. A las áreas clásicas desde donde se perfila la praxis evaluativa, como la pedagogía, la psicología del aprendizaje, la gestión escolar, la sociología o la estadística, la condición digital añade otras áreas de conocimiento como las matemáticas, la 
inteligencia artificial, la ingeniería de sistemas, el diseño, la comunicación mediada por tecnología, la bibliometría, entre otras, que vendrían a mejorar el planteamiento, desarrollo y representación de los procesos evaluativos y de sus resultados. Existen muchos avances, aunque sean rezagadas promesas todavía de tímida aplicación, que espolean el discurso y práctica de la evaluación en el campo educativo. Y más que referirnos aquí a sus concreciones, como si de un producto final se tratara, preferimos fijarnos en su capacidad de transformación desde su fundamento y una manera de hacerlo es abordar qué es lo que mejoran de manera sustantiva estas propuestas evaluativas apoyadas por lo digital. Ello nos lleva a plantear una suerte de análisis del campo de la evaluación y sugerir qué ámbitos de progreso son necesarios estimar.

Habiendo de elegir mejoras de esencia pero que sean a la vez posibles en un futuro próximo, nos fijamos en determinar ciertas características de lo que serían los entornos avanzados de evaluación y, en concreto, de la calidad del aprendizaje que constituye la única meta educativa. Entendemos que, en el marco de la sociedad digital, la digitalización de los procesos mediadores dirigidos a los profesores y los alumnos deberá materializarse en propuestas que lleguen a ofrecerse de manera altamente integrada, hecho que actualmente solo se deja entrever, de manera dispersa, y en contadas ocasiones.

Los entornos avanzados de evaluación digitalizada de los aprendizajes (lugar, estado o manera de proceder de un modo automatizado, fluido y efectivo mediado por las TIC) deberían no solo cumplir sino superar, en estos momentos, requisitos exigidos desde la investigación y la práctica educativas desde hace mucho tiempo y que todavía son recurrentes en informes actuales por su escasez (Tiven et al., 2018). Nos referimos a aspectos conocidos pero complejos de introducir de manera decidida en nuestras propuestas evaluativas en su globalidad y en los que la tecnología ha jugado un papel ambivalente ayudando, pero al mismo tiempo limitando, su puesta en el campo educativo.

Apuntamos a conocidos constructos que de la mano de la digitalización se tornan en verdaderos ámbitos de progreso en el ámbito de la evaluación educativa, como por ejemplo, el diseño explícito de la contextualización (evaluación auténtica); la apuesta por la complejidad cognitiva versus el resumen excesivo de contenidos (habilidades complejas, dejando atrás la linealidad delas evaluaciones eincluso el tiempo ajustado); el balance de los planteos de personalización y al mismo tiempo de socialización (superando la constrictiva individualización, abogando por la evaluación adaptativa avanzada y llegando a la supervisión de lo aprendido); evaluación formadora (aprender a evaluarse teniendo herramientas eficaces para ello: de representación, analíticas de aprendizaje, etc.); autorregulación y co-regulación (basada en el estudiante, no en el programa o profesor). En estos momentos se manifiestan avances sobre aspectos como el feedback oral, la corrección automatizada que puede llevar a la auto-puntuación y un uso más generalizado del big data y las analíticas de enseñanza y aprendizaje o el planteamiento de una evaluación educativa "smart". Todo ello para llegar a una presentación de entornos de evaluación más orgánicos 
que equilibre parte de las inversiones de trabajo docente y de los mismos sistemas educativos.

Como se puede ver, tanto la evaluación de la educación digital y la digitalización de la evaluación del aprendizaje no se reducen a la inserción de sistemas tecnológicos, sino a la comprensión pedagógica de su rol e impacto en los procesos educativos. Será necesario articular una comprensión que admita, como señala Bates (2018), que la evaluación del aprendizaje digital, como la calidad de la educación digital, aunque sea asistida de forma tecnológica no es una ciencia exacta.

Admitida la naturaleza inferencial de la evaluación por parte de profesores y también de estudiantes (Khine, 2018), la puesta en práctica de una agenda de progreso presenta una alta complejidad para el profesor; complejidad que mantiene un equilibrio irregular si se atiende al interés declarado muchas veces por los estudiantes y también, a la mejora cualitativa de los procesos de evaluación educativa con tecnología. No siendo garantía de buenos resultados, por lo menos a corto plazo, a nadie se le escapa que existe una, todavía, amplia brecha entre práctica y medida, a pesar de los considerables progresos realizados en el ámbito de los instrumentos de medición del aprendizaje, los recursos, el profesorado y la calidad en la esfera educativa. En este marco la tarea consiste en investigar, reflexionar y aplicar modelos que permitan ver de nuevo la educación desde la novedad digital.

Por ello, para la presentación global de los distintos artículos nos parece sugerente hacer el ejercicio de aplicar, entre todos, las dimensiones de lo simple, lo complicado, lo complejo y lo caótico apuntado en el marco conceptual Cynefin (Snowden, 2000). En nuestro caso esta práctica ayudaría a caracterizar, aunque de un modo genérico, la naturaleza de los cambios propuestos en el marco de la evaluación educativa acompañada de TIC que plantean los artículos seleccionados.

De manera muy resumida, en una realidad educativa diversa, compleja, conectada, adaptable y ahora un tanto caótica, lo simple abordaría problemáticas que identifican fácilmente sus causas y sus efectos, y también sus soluciones. En ese ámbito existe una respuesta correcta, conocida y compartida, aceptada más por costumbre que por indiscutible que en muchas ocasiones viene representada por las buenas prácticas en el campo de la evaluación. Lo complicado es lo que presentando muchos detalles supone una gran dificultad para ser entendido, explicado o resuelto, hecho que conlleva a distintas soluciones para un mismo problema, normalmente a través de técnicas más sofisticadas. Lo complejo se caracteriza por la combinación de distintos elementos muchas veces de distinta naturaleza, por lo que requiere análisis y experimentación, lo que desemboca en propuestas singulares. Lo caótico queda representado por prácticas evaluativas de frontera, donde no solo no hay soluciones estándar, sino que están al filo de la creación de nuevo conocimiento, hecho que no garantiza el éxito pero, eso sí, son fruto de una visión abierta e interdisciplinaria que supone una reacción rápida ante una realidad acuciante.

Es en esta línea de reflexión que caben los trabajos aportados en este monográfico que se presentan brevemente a continuación. Como se verá en los 
artículos la evaluación educativa tiene poco de simple, mucho de complejo y bastante de complicado; y no nos pronunciamos sobre lo caótico por falta de una teoría subyacente de definido interés y proyección, pero en la que nos hemos podido ver implicados en el contexto pandémico actual al tener que reaccionar, con carácter de urgencia, ante lo impredecible.

Para empezar, es en este marco más incierto generado por la pandemia que se pueden situar tres artículos del monográfico que hacen referencia a una evaluación de emergencia en el marco del COVID-19. Los trabajos son: Planificación, comunicación y metodologías activas: Evaluación online de la asignatura ingeniería de software durante la crisis del COVID-19, de Francisco José García-Peñalvo, Alicia García-Holgado y Andrea Vázquez-Ingelmo; Evaluación Remota de Aprendizajes en la Universidad: decisiones docentes para encarar un nuevo desafio, de Gisela Schwartzman, Carolina Roni, Michelle Berk, Erica Delorenzi, Mariana Sánchez, María Laura Eder y José Carlos Sánchez-Prieto; y Validación de una plataforma de evaluación formativa de la competencia digital docente en tiempos de Covid-19, de Virginia Viñoles-Cosentino, Francesc M. Esteve-Mon, María Ángeles Llopis-Nebot, Jordi Adell-Segura. Aunque cada uno de estos trabajos realiza una aportación sobre objetos de estudio evaluativo diferente, la constante en este grupo es el reto de comprender, de cara a tomar decisiones, sobre la normalización de la evaluación digital.

Otro segmento de trabajos lo pueden conformar los que estudian el feedback educativo. En un primer plano tenemos a: Procesos de feedback para fomentar la autorregulación con soporte tecnológico en la educación superior: Revisión sistemática, de Begoña Gros Salvat y Elena Cano y ¿̇Cómo implicar a los estudiantes para que utilicen el feedback online?, de Anna Espasa, Teresa Guasch. Ambos trabajos abordan la necesidad de plantear el reto evaluativo que supone el aprendizaje autorregulado con tecnología que, en buena cuenta, es una de las mayores esperanzas puesta en la potencialidad digital para favorecer un aprendizaje continuado a lo largo de la vida. En otro artículo, asociado al tema del refuerzo, titulado Peer Assessment as a Tool to Enhance Pre-Service Primary Bilingual Teachers' Training de Cristina Aránzazu Huertas-Abril, Francisco Javier Palacios-Hidalgo y María Elena GómezParra, se ahonda en la percepción de ese mismo mecanismo en el marco de la evaluación por pares en la formación inicial de los profesores de lenguas.

En la línea de evaluación formativa con tecnología, los artículos: La evaluación de la educación virtual: las e-actividades, de Julio Cabero-Almenara y Antonio PalaciosRodríguez, Rendimiento académico y deserción de estudiantes universitarios de un curso en modalidad virtual y presencial, de Eduardo Fabio Gonzales-López e Inés Evaristo Chiyong y una experiencia de diseño de una tarea de evaluación sumativa en formato transmedia para formación inicial de profesorado, de Linda Castañeda, ponen el foco de mira en la necesidad integrar el proceso de evaluación del aprendizaje virtual en el marco de la noción de e-actividades, gestionar pertinentemente la evaluación sumativa, resaltando aspectos cruciales sobre la función social de la 
evaluación como el rendimiento y el abandono, pero también aportan una reflexión y análisis de elementos sobre el diseño de contenidos digitales de las tareas cuanto eje vertebrador de las intenciones educativas.

Para cerrar el monográfico se presentan dos artículos relativos a la calidad educativa en la Educación Superior mediada por tecnología: Calidad en e-Learning: Un modelo para su evaluación en Educación Superior, de Alberto Ortiz-López, Susana Olmos-Migueláñez, José Carlos Sánchez-Prieto y Auditorías virtuales en el Programa de Sellos Internacionales de Calidad (SIC) de ANECA en tiempos de pandemia, de Ana Isabel Bonilla Calero, María Ángeles Serrano-García. La primera es de corte más institucional y focalizado en el estudio de dimensiones de calidad educativa del e-learning y el otro más centrado en el sistema educativo como tal atendiendo a los procesos de evaluación desde una agencia de calidad.

Por último, comentar que es interesante observar que no se han recibido propuestas de artículos sobre materiales, recursos o dispositivos digitales referidos a la evaluación como se podía esperar en una convocatoria abierta en la que la digitalización ocupa un espacio importante. Existen muchas posibles explicaciones y, sin obviar ninguna de ellas, los editores de este monográfico se decantan por pensar que estos aspectos más técnicos se encuentran entre "lo simple", que sería lo mismo que decir que son realidades que, a pesar de su naturaleza cambiante, se perciben abordables y solucionables. Desde esta visión, se apunta a que la tecnología en sí misma, no preocupa excesivamente, como tampoco los contenidos educativos por ser los profesores-investigadores dominantes del conocimiento que imparten. Pero sí que se refleja que existe todo un diálogo de interés entre lo complejo y lo complicado siendo clara protagonista la metodología docente, evaluativa en este caso, en la manera de enseñar-evaluar el aprendizaje de los estudiantes en un marco de calidad evaluativa digital.

\section{REFERENCIAS}

ANECA (2020). Estrategia de ANECA para el aseguramiento de la calidad en la enseñanza virtual. http://www. aneca.es/Sala-de-prensa/Noticias/2020/ Estrategia-de-ANECA-para-elaseguramiento-de-la-calidad-en-laensenanza-virtual

Bates, A. T. (2018). Teaching in a digital age: Guidelines for designing teaching and learning. Tony Bates Associates.

Hodges, C., Moore, S., Lockee, B., Trust, T., y Bond, A. (2020). The difference between emergency remote teaching and online learning. Educause Review, 27. https:// er.educause.edu/articles/2020/3/the- difference-between-emergency-remoteteaching-and-online-learning

Khine, M. S. (2018). International trends in Educational Assessment: Issues and Practice. Bill Publishing. https://doi. org/10.1163/9789004393455

Snowden, D. (2000). Cynefin: A sense of time and space, the social ecology of knowledge management. En C. Despres y D. Chauvel (Eds.), Knowledge Horizons: The Present and the Promise of Knowledge Management. Butterworth Heinemann. https://doi.org/10.1016/B978-0-75067247-4.50015-X 
Tiven, M. B., Fuchs, E. R., Bazari, A., y MacQuarrie, A. (2018). Evaluating Global Digital Education: Student Outcomes
Framework. Bloomberg Philanthropies and the Organisation for Economic Cooperation and Development.

\section{PERFIL ACADÉMICO Y PROFESIONAL DE LOS AUTORES}

Elena Barberà Gregori. Doctora en Psicología por la Universitat de Barcelona. Catedrática de Psicología Educativa, actualmente es la directora del programa del doctorado en Educación y TIC en la Universidad Oberta de Catalunya. Sus áreas de investigación son regulación educativa, evaluación del aprendizaje en línea e interacción educativa.

E-mail: ebarbera@uoc.edu

\section{DIRECCIÓN DE LA AUTORA}

Estudios de Psicología y Ciencias de la Educación

Universitat Oberta de Catalunya

Rambla del Poble Nou, 156

o8018 Barcelona

Cristóbal Suárez-Guerrero. Doctor en Educación, procesos de formación en espacios virtuales por la Universidad de Salamanca y profesor del Departamento de Didáctica y Organización Escolar de la Universitat de València. Sus áreas de investigación y desarrollo educativo son pedagogía digital, cultura educativa digital, competencia digital e innovación didáctica con tecnología.

E-mail: cristobal.suarez@uv.es

DIRECCIÓN DEL AUTOR

Facultat de Magisteri

Universitat de València

Av. Tarongers, 4 - 46022

València - España

Fecha de recepción del artículo: 16/03/2021

Fecha de aceptación del artículo: 16/03/2021

Fecha de aprobación para maquetación: 18/03/2021 